\title{
DYNAMICS FOR VORTEX CURVES OF THE GINZBURG-LANDAU EQUATIONS
}

\author{
LIU ZUHAN
}

\begin{abstract}
We study the asymptotic behaviour of solutions to the evolutionary Ginzburg- Landau equations in three dimensions. We show that the motion of the Ginzburg-Landau vortex curves is the flow by curvature.
\end{abstract}

\section{INTRODUCTION}

Let $Q=\Omega \times[0, l], \Omega \subset R^{2}$, be a bounded smooth domain, $g: \Sigma=\partial \Omega \times[0, l] \rightarrow S^{1}$ a $C^{1, \alpha}$-map such that $\operatorname{deg}\left(g, \partial \Omega_{z}\right)=d>0$ for all $0 \leqslant z \leqslant l$. Here $\Omega_{z}=\Omega \times\{z\}$. Let $a: Q \rightarrow R$ be a smooth function (say $C^{3}(\bar{Q})$ ) with positive lower bound.

We consider the following problem:

$$
\begin{aligned}
\frac{\partial u_{\varepsilon}}{\partial t} & =\frac{1}{a(x)} \operatorname{div}(a(x) \nabla u)+\frac{1}{\varepsilon^{2}} u_{\varepsilon}\left(1-\left|u_{\varepsilon}\right|^{2}\right) \quad \text { in } Q \times R_{+}, \\
u_{\varepsilon}(x, 0) & =u_{\varepsilon}^{0}(x), \quad x \in Q, \\
u_{\varepsilon}(x, t) & =g(x), \quad x \in \Sigma, t \geqslant 0, \\
\frac{\partial u_{\varepsilon}}{\partial z} & =0 \quad \text { for } z=0, l,
\end{aligned}
$$

where $u_{\varepsilon}: Q \times R_{+} \rightarrow R^{2}$. The system (1.1)-(1.4) can be viewed as a simplified evolutionary Ginzburg-Landau equation in the theory superconductivity of inhomonogence.

The aim of this article is to understand the dynamics of vortices, or zeros, of solutions $u$ of (1.1)- (1.4). Its importance to the theory of superconductivity and applications are addressed in many earlier papers $[4,9,10,11,14]$.

Let $\Gamma_{0}$ be a collection of $d$ embedded $C^{2}$-curves in $Q$ with $\partial \Gamma_{0} \subset \Omega \times\{0, l\}$. Moreover, we assume $\Gamma_{0}$ intersects $\Omega \times\{0, l\}$ orthogonally along $\partial \Gamma_{0}$. Note that the last assumption is compatiable with the assumption $\frac{\partial u_{\varepsilon}^{0}}{\partial z}=0$ for $z=0, l$. (That is the natural compatibilty condition for problem (1.1)-(1.4). Similarly, we also assume that $u_{\varepsilon}^{0}=g$ on $\Sigma$.)

For the initial data $u_{\varepsilon}^{0}$, we make the following assumptions:

\section{Received 2nd May, 2000}

This work supported by the National Nature Science Foundation of China (10071067).

Copyright Clearance Centre, Inc. Serial-fee code: 0004-9727/01 \$A2.00+0.00. 
(H1) $\int_{Q} \rho^{2}(x)\left[\left|\nabla u_{\varepsilon}^{0}\right|^{2}+\left(\left|u_{\varepsilon}^{0}\right|^{2}-1\right) /\left(2 \varepsilon^{2}\right)\right] d x \leqslant K$ for all $0<\varepsilon \leqslant 1$. Here $\rho(x)=\operatorname{dist}\left(x, \Gamma_{0}\right)$;

(H2) $u_{\varepsilon}^{0}$ converges as $\varepsilon \rightarrow 0^{+}$in the $C^{0}$-norm away from $\Gamma_{0}$ to a map $u^{0}$ with its image in $S^{1}$;

(H3) Let $\Gamma_{0}^{i}, i=1, \cdots, k$, be connected components of $\Gamma_{0}$, and let $\delta>0$ be chosen so that the sets $\Gamma_{0}^{i}(\delta), i=1, \cdots, k$, are pairwise disjoint. Here $\Gamma_{0}^{i}(\delta)=\left\{x \in Q: \operatorname{dist}\left(x, \Gamma_{0}^{i}\right) \leqslant \delta\right\}$.

Let $T>0$, and $\left\{\Gamma_{t}\right\}, 0 \leqslant t \leqslant T$, be a family of embedded $C^{2}$-curves inside $Q$ with boundaries $\left\{\partial \Gamma_{t}\right\}$ contained in $\Omega \times\{0, l\}$, assume $\Gamma_{t}$ intersects with $\Omega \times\{0, l\}$ orthogonally along $\partial \Gamma_{t}$, which are obtained from $\Gamma_{0}$ by the following equations in $R^{3}$ :

$$
\left\{\begin{array}{l}
\frac{d x(p, t)}{d t}=\vec{H}(x(p, t), t)-\pi \frac{\nabla a}{a}(x(p, t)), \\
x(p, 0)=p \in \Gamma_{0}
\end{array}\right.
$$

where $\pi$ is the projection onto the normal space of $\Gamma_{t}$, and the curvature vector $\vec{H}$ of $\Gamma_{t}$ is characterised by the property

$$
\int_{\Gamma_{t}} \operatorname{div}^{\Gamma_{t}} \phi d \mathcal{H}^{1}=-\int_{\Gamma_{t}} \vec{H} \cdot \phi d \mathcal{H}^{1}, \quad \forall \phi \in\left(\phi_{1}, \phi_{2}, \phi_{3}\right) \in C^{1}\left(R^{3}, R^{3}\right),
$$

here $\operatorname{div}^{\Gamma_{t}} \phi=d_{i}^{\Gamma_{t}} \phi_{i}$ is the tangential divergence of $\phi[12]$. In the case $a=1$, equation (1.5) denotes the flow by mean curvature with codimension 2 in $R^{3}$.

THEOREM 1.1. Assume that $a \in C^{3}(\bar{Q})$ and $a_{0}=\min _{\bar{Q}} a>0$. Under assumptions (H1)-(H3) and for each $t, 0 \leqslant t \leqslant T$, one has (by taking subsequences if necessary) that $u_{\varepsilon}(x, t) \rightarrow u_{*}(x, t)$ weakly in $H_{\text {loc }}^{1}\left(\bar{Q} \backslash \Gamma_{t}\right)$. Here $u_{*}(x, t)$ satisfies:

$$
\partial_{t} u_{*}-\frac{1}{a} \operatorname{div}\left(a \nabla u_{*}\right)=\left|\nabla u_{*}\right|^{2} u_{*} \text { in } Q \backslash \Gamma_{\mathfrak{t}}
$$

Now we briefly describe some mathematical advances concerning this problem. In two space dimensions, $a=1$, the dynamical law for vortices was formally derived in $[8,14]$. The first rigorous mathematical proof of this dynamical law, which is of the form $\frac{d}{d t} x(t)=-\nabla w(x(t))$, was given by Lin in $[4,5]$. See also $[6$, Lecture 3$]$. In $[4,5]$, one allows vortices of degree \pm 1 and assumes that they have the same sign. For vortices of degree \pm 1 (possibly of different signs), the same type of dynamical law has recently been shown [3]. We refer to [7] for vortex dynamics under the Neumann boundary conditions for pinning conditions. In three space dimensions, $a=1$, a similar dynamical law was also established in [7] for nearly parallel filaments. The short-time dynamical law for codimension 2 interfaces in higher dimensions was shown in [7]. In two space dimensions, $a \not \equiv 1$, the dynamical law was established in [7].

The rest of the paper is organised as follows. In Section 2, we collect some basic facts on the curve flow. In Section 3, we prove the weak convergence. 


\section{Mean CuRvature flow With CODimension 2}

Given a set $E \subset R^{3}$, we set

$$
\eta_{E}(x)=\frac{1}{2}(\operatorname{dist}(x, E))^{2} .
$$

The following results on the square distance function have been proved in [6]. Let $\gamma$ be a smooth embedded curve in $R^{3}$; then $\eta_{\gamma}$ is smooth in a suitable tubular neighbourhood $\Omega$ of $\gamma .-\Delta \nabla \eta_{\gamma}$ coincides, on $\gamma$, with the curvature vector $\vec{H}$ of $\gamma$.

Lemma 2.1. [2, Lemma 3.7] Let $\left(\Gamma_{t}\right)_{t \in[0, T]}$ be a smooth fow. Then there exists $\sigma>0$ such that the function

$$
\eta(x, t):=\frac{1}{2} \operatorname{dist}^{2}\left(x, \Gamma_{t}\right)
$$

is smooth in $\left\{(x, t) \in R^{3} \times[0, T]: \eta \leqslant \sigma\right\}$. Moreover, the displacement of the flow is given by

$$
\frac{d x(p, t)}{d t}=-\nabla \eta_{t}(x(p, t), t), \quad \forall t \in[0, T], p \in \Gamma_{0} .
$$

In particular, $\left(\Gamma_{t}\right)_{t \in[0, T]}$ is a smooth curvature flow defined by (1.5) if and only if

$$
\nabla \eta_{t}=\Delta \nabla \eta-\nabla^{2} \eta \frac{\nabla a}{a}, \text { on } \Gamma_{t} .
$$

Short time existence for curvature flow of smooth initial space curves is a consequence of a general theorem proved in $[\mathbf{1}, \mathbf{1 3}]$.

Lemma 2.2. Assume that $\gamma_{0}$ is a embedded $C^{2}$-curve in $Q$ with $\partial \gamma_{0} \in \Omega \times\{0, l\}$. Assume $\Gamma_{0}$ intersects $\Omega \times\{0, l\}$ orthogonally along $\partial \gamma_{0}$. Then there exist a positive number $t_{0}>0$ and a family of embedded $C^{2}$-curves inside $Q$ with $\partial \Gamma_{t} \subset \Omega \times\{0, l\}$ such that the following system of equalities holds on $\gamma_{t}$ :

$$
\frac{\partial \nabla \eta_{\gamma}}{\partial t}(t, p)-\Delta \nabla \eta_{\gamma}(t, p)+\nabla^{2} \eta \frac{\nabla a}{a}(p)=0, \quad t \in\left[0, t_{0}\right], p \in \gamma_{t},
$$

and $\gamma_{t}$ intersects with $\Omega \times\{0, l\}$ orthogonally along $\partial \gamma_{t}$.

\section{THE PROOF OF THEOREM 1.1}

LEMMA 3.1. (Uniformly estimate)

$$
\int_{0}^{T} \int_{Q \backslash \Gamma_{t}(\delta)}\left[\left|u_{\varepsilon t}\right|^{2}+\frac{1}{2} a(x)\left(\left|\nabla u_{\varepsilon}\right|^{2}+\frac{1}{2 \varepsilon^{2}}\left(1-\left|u_{\varepsilon}\right|^{2}\right)^{2}\right)\right] d x d t \leqslant C(\delta, T, \sigma),
$$

where $\sigma>0$ is such that the sets $\Gamma_{t}^{i}(4 \sigma), i=1,2, \cdots, k$, are pairwise disjoint for all $0 \leqslant \Gamma, 0<\delta \leqslant \sigma$. Here $\Gamma_{t}^{i}(4 \sigma)=\left\{x \in Q:\right.$ dist $\left.\left(x, \Gamma_{t}^{i}\right) \leqslant 4 \sigma\right\}$. 
PROOF: Let $\phi_{\sigma}: R_{+} \rightarrow R_{+}$be a smooth monotone function such that

$$
\phi_{\sigma}(r)=\left\{\begin{array}{ll}
r^{2} & \text { if } \quad r \leqslant \sigma \\
4 \sigma^{2} & \text { if } \quad r \geqslant 2 \sigma .
\end{array} \quad(\sigma>0) .\right.
$$

Define

$$
\rho(x, t)=\operatorname{dist}\left(x, \Gamma_{t}\right)
$$

Assume that

$$
\min \left\{|x-y|: x \in \Gamma_{t}, y \in \sum, 0 \leqslant t \leqslant \Gamma\right\} \geqslant 4 \sigma .
$$

Using integration by parts, one gets

$$
\begin{aligned}
\frac{d}{d t} \int_{Q} \frac{1}{2} \phi_{\sigma}(\rho(x, t)) a(x)\left[|\nabla u|^{2}+\frac{1}{2 \varepsilon^{2}}\left(1-|u|^{2}\right)^{2}\right] \\
=\int_{Q} \frac{1}{2}\left(\frac{d}{d t} \phi_{\sigma}\right) \cdot a(x)\left[|\nabla u|^{2}+\frac{1}{2 \varepsilon^{2}}\left(1-|u|^{2}\right)^{2}\right] \\
\quad+\int_{Q} \phi_{\sigma} a\left[\nabla u \cdot \nabla u_{t}+\frac{1}{2 \varepsilon^{2}}\left(1-|u|^{2}\right) \cdot\left(-2 u u_{t}\right)\right] \\
=: I+I I .
\end{aligned}
$$

We shall set $\phi_{\sigma}=\phi, \quad u_{\varepsilon}=u$.

$$
\begin{aligned}
I I & =\int_{Q} \phi\left[-\nabla(a \nabla u)-\frac{a(x)}{\varepsilon^{2}}\left(1-|u|^{2}\right) u\right] u_{t}-\int_{Q} \nabla \phi \cdot a \cdot \nabla u \cdot u_{t} \\
& =-\int_{Q} \phi\left|u_{t}\right|^{2}-\int_{Q} a \nabla \phi \nabla u \cdot u_{t} .
\end{aligned}
$$

Now we calculate the expression $a \nabla \phi \nabla u \cdot u_{t}$. We shall use the summation convention, and simplify notation.

$$
\begin{aligned}
a \nabla \phi \nabla u \cdot u_{t} & =\nabla \phi \nabla u\left[\operatorname{div}(a \nabla u)+\frac{1}{\varepsilon^{2}} a \cdot\left(1-|u|^{2}\right) u\right] \\
& =\left(a u_{j}\right)_{j} u_{i} \phi_{i}+\frac{1}{\varepsilon^{2}} a\left(1-|u|^{2}\right) u \cdot u_{i} \phi_{i} \\
& =\left(a u_{i} u_{j}\right)_{j} \phi_{i}-a u_{j} u_{i j} \phi_{i}-\left[\frac{1}{4 \varepsilon^{2}} a\left(1-|u|^{2}\right)^{2}\right]_{i} \phi_{i}+\frac{1}{4 \varepsilon^{2}}\left(1-|u|^{2}\right)^{2} a_{i} \phi_{i} \\
& =\left(a u_{i} u_{j}\right)_{j} \phi_{i}-\frac{1}{2}\left(\left|u_{j}\right|^{2}\right)_{i} \phi_{i}-\left[\frac{1}{4 \varepsilon^{2}} a\left(1-|u|^{2}\right)^{2}\right]_{i} \phi_{i}+\frac{1}{4 \varepsilon^{2}}\left(1-|u|^{2}\right)^{2} a_{i} \phi_{i} .
\end{aligned}
$$

Hence

$$
\begin{gathered}
\int_{Q} a \nabla \phi \nabla u \cdot u_{t}=\int_{Q}-a \phi_{i j} u_{i} u_{j}+\frac{1}{2} \Delta \phi \cdot a|\nabla u|^{2}+\frac{1}{2} \nabla a \cdot \nabla \phi \cdot|\nabla u|^{2} \\
+\Delta \phi \cdot \frac{1}{4 \varepsilon^{2}} a\left(1-|u|^{2}\right)^{2}+\frac{1}{4 \varepsilon^{2}}\left(1-|u|^{2}\right)^{2} \cdot \nabla a \cdot \nabla \phi \\
=-\int_{Q} a \phi_{i j} u_{i} u_{j}+\int_{Q} \Delta \phi \cdot \frac{1}{2} a\left[|\nabla u|^{2}+\frac{1}{2 \varepsilon^{2}}\left(1-|u|^{2}\right)^{2}\right] \\
+\int_{Q} \frac{\nabla a}{a} \cdot \nabla \phi \cdot \frac{1}{2} a\left[|\nabla u|^{2}+\frac{1}{2 \varepsilon^{2}}\left(1-|u|^{2}\right)^{2}\right]
\end{gathered}
$$


So, we have

$$
\begin{aligned}
\frac{d}{d t} \int_{Q} \phi \frac{1}{2} a\left[|\nabla u|^{2}\right. & \left.+\frac{1}{2 \varepsilon^{2}}\left(1-|u|^{2}\right)^{2}\right] \\
=\int_{Q}\left[\phi_{t}-\Delta \phi-\frac{\nabla a}{a} \cdot \nabla \phi\right] & \frac{1}{2} a\left[|\nabla u|^{2}+\frac{1}{2 \varepsilon^{2}}\left(1-|u|^{2}\right)^{2}\right] \\
& +\int_{Q} a \phi_{i j} u_{i} u_{j}-\int_{Q} \phi\left|u_{t}\right|^{2}
\end{aligned}
$$

Next we observe that on the set $\{x \in Q: \rho(x, t)<\sigma\}, \quad\left(\phi_{i j}\right) \leqslant I$ in the sense that

$$
\phi_{i j} \xi_{i} \xi_{j} \leqslant|\xi|^{2} \quad \text { for all } \xi \in R^{3} \text {. }
$$

Also, on $\Gamma_{t}$, we have $\phi_{t}=0, \Delta \phi=0$. Since $\Gamma_{t}$ is obtained from $\Gamma_{0}$ by curvature flow (1.5), by Lemma 2.1, we have

$$
\nabla\left(\phi_{t}-\Delta \phi-\frac{\nabla a}{a} \cdot \nabla \phi\right)=0 \quad \text { on } \Gamma_{t} .
$$

Thus

$$
\begin{aligned}
\phi_{t}-\Delta \phi-\frac{\nabla a}{a} \cdot \nabla \phi & \leqslant-2+C_{0} \cdot \rho^{2}(x, t) \\
& =-2+C_{1} \phi .
\end{aligned}
$$

Combining (3.9) and (3.12) with the fact that $\left(\phi_{i j}\right) \leqslant I$, we have

$$
\frac{d}{d t} \int_{Q} \frac{1}{2} \phi \cdot a\left[|\nabla u|^{2}+\frac{1}{2 \varepsilon^{2}}\left(1-|u|^{2}\right)^{2}\right] \leqslant C \int_{Q} \phi \frac{1}{2} a\left[|\nabla u|^{2}+\frac{1}{2 \varepsilon^{2}}\left(1-|u|^{2}\right)^{2}\right] .
$$

Now we use Gronwall's inequality and the assumption (H1) to obtain

$$
\sup _{0 \leqslant t \leqslant T} \int_{Q} \phi_{\sigma}(\rho(x, t)) \frac{1}{2} a\left[|\nabla u|^{2}+\frac{1}{2 \varepsilon^{2}}\left(1-|u|^{2}\right)^{2}\right] d x \leqslant C(\sigma, T, K) .
$$

The last inequality implies that

$$
\int_{Q \backslash \Gamma_{t}(\delta)} \frac{1}{2} a\left[|\nabla u|^{2}+\frac{1}{2 \varepsilon^{2}}\left(1-|u|^{2}\right)^{2}\right] d x \leqslant C(\delta, \sigma, T, K),
$$

for all $0 \leqslant t \leqslant T$ and $0<\varepsilon<<1$. 
Next, for $0 \leqslant t_{1} \leqslant t \leqslant t_{2} \leqslant T$, we let $\eta(x)$ be a smooth cutoff function supported in $Q \backslash \bigcup_{t_{1} \leqslant t \leqslant t_{2}} \Gamma_{t} ;$ then

$$
\begin{aligned}
\frac{d}{d t} \int_{Q} & \eta^{2}(x) \frac{1}{2} a\left[|\nabla u|^{2}+\frac{1}{2 \varepsilon^{2}}\left(1-|u|^{2}\right)^{2}\right] d x \\
& =\int_{Q} \eta^{2} a\left[\nabla u \cdot \nabla u_{t}-\frac{1}{\varepsilon^{2}}\left(1-|u|^{2}\right) u \cdot u_{t}\right] \\
& =-\int_{Q} \eta^{2}\left[\nabla(a \nabla u)+\frac{1}{\varepsilon^{2}}\left(1-|u|^{2}\right) u\right] u_{t}-2 \int_{Q} a \eta \nabla \eta \cdot \nabla u \cdot u_{t} \\
& =-\int_{Q} \eta^{2}(x)\left|u_{t}\right|^{2}-2 \int_{Q} a \eta \nabla \eta \nabla u \cdot u_{t} \\
& \leqslant-\frac{1}{2} \int_{Q} \eta^{2}(x)\left|u_{t}\right|^{2}+C \int_{Q}|\nabla \eta|^{2}|\nabla u|^{2}
\end{aligned}
$$

From (3.15) and (3.16), we obtain that

$$
\left\|u_{\epsilon}\right\|_{H_{l o c}^{1}\left(\bar{Q} \times[0, T] \backslash \bigcup_{0 \leqslant t \leqslant T} \Gamma_{t}\right)} \leqslant C
$$

The proof of Lemma 3.1 is completed.

Hence, by taking a subseqence if necessary, we have

$$
u_{\varepsilon} \rightarrow u_{*} \quad \text { weakly in } \quad H_{l o c}^{1}\left(\bar{Q} \times[0, T] \backslash \bigcup_{0 \leqslant t \leqslant T} \Gamma_{t}\right) .
$$

It is easy to verify that $u_{*}$ satisfies

$$
\frac{\partial u_{*}}{\partial t}=\frac{1}{a} \operatorname{div}\left(a \nabla u_{*}\right)+u_{*}\left|\nabla u_{*}\right|^{2} \quad \text { in } H_{l o c}^{1}\left(\bar{Q} \times[0, T] \backslash \bigcup_{0 \leqslant t \leqslant T} \Gamma_{t}\right) .
$$

The proof of Theorem 1.1 is completed.

\section{REFERENCES}

[1] S. Altschuler, 'Singularities of the curve shortening flow for space curves', J. Differential Geom. 34 (1991), 491-514.

[2] L. Ambrosio and H.M. Soner, 'Level set approach to mean curvature flow in arbitrary codimension', J. Differental Geom. 43 (1996), 693-737.

[3] R. Jerrard and M. Soner, 'Dynamics of Ginzburg-Landau vortices', Arch. Rational Mech. Anal. 142 (1998), 99-125..

[4] F.H. Lin, 'Some dynamical properties of Ginzburg-Landau vortices', Comm. Pure Appl. Math. 49 (1996), 323-359.

[5] F.H. Lin, 'A remark on the previous paper: "Some dynamical properties of Ginzburg-Landau vortices"', Comm. Pure Appl. Math. 49 (1996), 361-364. 
[6] F.H. Lin, 'Static and moving vortices in Ginzburg-Landau theories', in Nonlinear partial differential equations in geometry and physics (Knoxville, TN, 1995), Progr. Nonlinear Differential Equations Appl. 29 (Birkhauser, Basel, 1997), pp. 71-111.

[7] F.H. Lin, 'Complex Ginzburg-Landau equations and dynamics of vortices, filaments, and codimension-2 submanifolds', Comm. Pure Appl. Math. 51 (1998), 385-441.

[8] J.C. Neu, 'Vortices in complex scalar fields', Phys. D. 43 (1990), 385-406.

[9] J.C. Neu, 'Vortex dynamics of the nonlinear wave equation', Phys. D. 43 (1990), 407-420.

[10] L. Peres and J. Rubinstein, 'Vortex dynamics in U(1) Ginzburg-Landau models', Phys. D. 64 (1993), 299-309.

[11] J. Rubinstein, 'On the equilibrium position of Ginzburg-Landau vortices', $Z$. Angew. Math. Phys. 46 (1995), 739-751.

[12] L. Simon, Lectures on geometric measure theory (Centre for Math. Analysis, Australian National University, A.C.T., 1984).

[13] A. Stahl, 'Regularity estimates for solutions to the mean curvature flow with a Neumann boundary condition', Calc. Var. Partial Differential Equations 4 (1996), 385-407.

[14] E. Weinan, 'Dynamics of vortices in Ginzburg-Landau theories with applications to supconductivity', Phys. D. 77 (1994), 383-404.

Department of Mathematics

Normal College

Yangzhou University

Yangzhou 225002

China

e-mail: zuhanl@yahoo.com 